\section{SGLT-2 Inhibitors: Fighting Cardiovascular Disease Mortality in Diabetes}

Sir,

Cardiovascular (CV) events are the leading cause of mortality in diabetic patients. Managing this macrovascular complication has been the forefront concern of both endocrinologists and cardiologists. Since the UK Prospective Diabetes study showed that better glycemic control improves CV outcomes in newly diagnosed diabetes patients, 1 what followed from here was an immense number of trials trying to find the best possible outcome for such patients. However, in early 2008, ACCORD (Action to Control Cardiovasuclar Risk in Diabetes) study debunked this aggressive approach to maintain a tighter glycemic control, as it may lead to an increased risk of death due to hypoglycemia. ${ }^{2}$

The hastened approval of TZDs by the FDA to treat diabetes mellitus (DM) patients further accelerated this calamity in terms of increased deaths due to heart failure and myocardial infarction (MI). It provoked them to design trials in such a way as to lower the glycemic index without compromising the CV outcome. In the past decade, we have seen novel drugs entering the realm of good glycemic control, increased rate of $\mathrm{HbA} 1 \mathrm{c}$ reduction, obesity-treatment and $\mathrm{CV}$ and renoprotective qualities. These new drug classes are the glucagon like peptide-1 (GLP-1) agonists and the sodium-glucoselinked-transporter-2 (SGLT-2) inhibitors.

The CANVAS (Canagliflozin Cardiovascular Assessment Study) programme compared efficacy of canagliflozin with placebo in diabetics who had an elevated risk of $\mathrm{CV}$ disease. The primary outcome of the study was focused on a combination of $\mathrm{CV}$ death, nonfatal $\mathrm{MI}$, or nonfatal stroke.

The trial concluded a significant decrease in the incidence of the primary outcome event in the canagliflozin group: 26.9 vs. 31.5 participants with an event per 1000 patientyears (hazard ratio, $0.86 ; 95 \% \mathrm{Cl}, 0.75$ to $0.97 ; p<0.001$ for noninferiority; $p=0.02$ for superiority). The only serious adverse effect seen in the group of patients taking canagliflozin was a higher risk of amputation of toes, feet, or legs; and was observed more in patients who had a history of peripheral vascular disease or a previous amputation. ${ }^{3}$

The CANVAS-R trial was a subset of the CANVAS programme and had the primary objective to study the effect of cangliflozin on kidney disease progression, with similar study design to the CANVAS trial. ${ }^{4}$ It concluded that progression of albuminuria occurred less frequently with canagliflozin. ${ }^{3}$ This finding can possibly be attributed to the nephro-protective effects of SGLT-2 inhibitors. By increasing angiotensin, they can restore the intra glomerular pressure, thus demonstrating vasodilatory and anti-inflammatory properties. ${ }^{5}$

These findings from the CANVAS programme have played a significant role in evolving our understanding of the effects of canagliflozin, and the broader SGLT2 inhibitor class, on a variety of efficacy and safety outcomes of crucial standing to patients with diabetes. 4

The SGLT-2 class is coming up to be the front runner in managing micro- and macro-vascular complications along with good glycemic control. Due to its non-insulin effect, it can be paired up with insulin or other insulin secretagogues to further improve $\mathrm{HbA1c}$. These are new horizons in fighting secondary complications of diabetes. Further trials are anticipated which should focus on all drugs of this class with a homogenous inclusion criteria of patients, similar risk factors, and a standardised outcome, addressing differences in race, pre-existing vascular, cardiac and renal conditions, and a longer duration of follow-up to obtain the most accurate results.

\section{REFERENCES}

1. Intensive blood-glucose control with sulphonylureas or insulin compared with conventional treatment and risk of complications in patients with type 2 diabetes (UKPDS 33). Lancet 1998; 352: 837-53.

2. Gerstein HC, Miller ME, Byington RP, Goff DC Jr., Bigger JT, Buse JB, et al. Effects of intensive glucose lowering in type 2 diabetes. New Engl J Med 2008; 358:2545-59.

3. Neal B, Perkovic V, Mahaffey K, de Zeeuw D, Fulcher G, Erondu $\mathrm{N}$, et al. Canagliflozin and cardiovascular and renal events in type 2 diabetes. New Engl J Med 2017; 377:644-57.

4. Neal B, Perkovic V, Mahaffey KW, Fulcher G, Erondu N, Desai M, et al. Optimizing the analysis strategy for the CANVAS Program: a prespecified plan for the integrated analyses of the CANVAS and CANVAS-R trials. Diabetes Obes Metab 2017; 19:926-35.

5. Natalia G, Vallianou, Geladari E, Kazazis CE. SGLT-2 inhibitors: Their pleiotropic properties. Diabetes Metab Syndr Clin Res Rev 2017; 11:311-5.

Zunaira Abdul Ghaffar, Shahzad Anwar and Syed Wajih Rizvi

1 Department of Medicine, Dow Medical College, Dow University of Health Sciences, Karachi, Pakistan

2 University of Medicine and Dentistry, New Jersey, USA

Correspondence: Dr. Zunaira Abdul Ghaffar, Tulip Street, Gulistan-e-Johar Block 14, Karachi, Pakistan

E-mail: zunairag@hotmail.com

Received: March 31, 2018; Accepted: September 29, 2018 